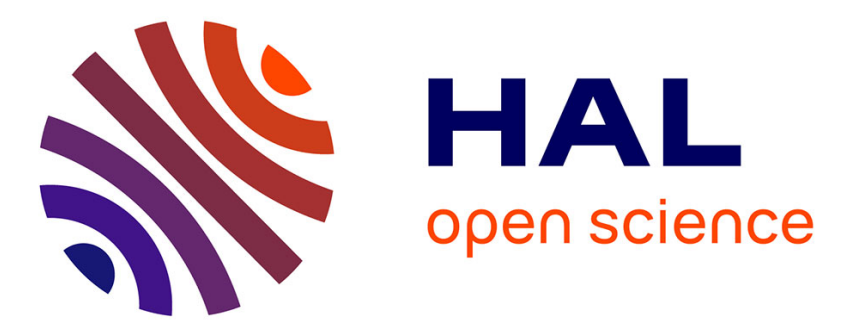

\title{
Coupled x-ray high-speed imaging and pressure measurements in a cavitating backward facing step flow
}

\author{
G. Maurice, Nathanaël Machicoane, S. Barre, H. Djeridi
}

\section{To cite this version:}

G. Maurice, Nathanaël Machicoane, S. Barre, H. Djeridi. Coupled x-ray high-speed imaging and pressure measurements in a cavitating backward facing step flow. Physical Review Fluids, 2021, 6 (4), pp.044311. 10.1103/PhysRevFluids.6.044311 . hal-03210205

\section{HAL Id: hal-03210205 \\ https://hal.science/hal-03210205}

Submitted on 27 Apr 2021

HAL is a multi-disciplinary open access archive for the deposit and dissemination of scientific research documents, whether they are published or not. The documents may come from teaching and research institutions in France or abroad, or from public or private research centers.
L'archive ouverte pluridisciplinaire HAL, est destinée au dépôt et à la diffusion de documents scientifiques de niveau recherche, publiés ou non, émanant des établissements d'enseignement et de recherche français ou étrangers, des laboratoires publics ou privés. 


\title{
Coupled x-ray high-speed imaging and pressure measurements in a cavitating backward facing step flow
}

\author{
G. Maurice $\odot,{ }^{1}$ N. Machicoane $\odot,{ }^{2}$ S. Barre,${ }^{2}$ and H. Djeridi ${ }^{2}$ \\ ${ }^{1}$ Hydroquest Society, 38240 Grenoble, France \\ ${ }^{2}$ Laboratoire des Ecoulements Géophysiques et Industriels, Univ. Grenoble Alpes, CNRS, \\ Grenoble INP, LEGI, 38000 Grenoble, France
}

(Received 29 October 2020; accepted 31 March 2021; published 27 April 2021)

\begin{abstract}
The purpose of the present experimental study is to get a better understanding of the dynamics of the vapor phase spatiotemporal repartition in a cavitating backward facing step flow. We provide a refined data base of the use of the void fraction transport equation to model such flows. The backward facing step flow provides a well-known test case to compare vortex dynamics between single and two-phase flow. To evidence the vapor phase dynamics, the flow is probed by high-speed $\mathrm{x}$-ray attenuation techniques and by pressure fluctuation measurements at the walls. Long-time dynamics are also visualized using conventional high-speed imaging synchronized with pressure measurements. Large vortex structures, free shear layer instability, wall interaction and reverse flow are observed. The two-phase structures are studied at different cavitation levels corresponding void fractions ranging from $1 \%$ to $50 \%$. The topology of the mean and fluctuating void fraction maps is performed, leading to the establishment of three specific areas in the flow. These areas are distinguished by the underlying mechanisms happening within them: vaporization, transport, and condensation. The statistical analysis underlines the existence of extreme events associated with high void fraction levels and wave propagations. While these events are associated with topological changes from a shear layer to a wake mode that also exist in the single-phase case, they are associated with much lower frequency at high cavitation levels.
\end{abstract}

DOI: 10.1103/PhysRevFluids.6.044311

\section{INTRODUCTION}

The present study is conducted within the framework of research on cavitation for space turbopump inducer applications. The main objective is to analyze cavitation effects which can occur when the local pressure of the liquid is lower than the vapor pressure. This phenomenon causes erosion damages, vibrations, noise, and performance damping. Indeed, in the case of inducer, the vapor phase appears on the suction side of the blades, as attached cavities (stable or pulsating depending on the operating conditions), and inside the gap between blade's tip and the housing, where the fluid is highly sheared. The latter region corresponds to separating/reattaching flow, whose behavior is reflected in the canonical backward-facing step flow. Such flow presents a mixing shear layer associated with the development of Kelvin-Helmholtz instabilities, with vorticity regions corresponding to low-pressure areas and large-scale coherent structures near the reattaching region. The latter are known to induce wall-pressure fluctuations [1-3]. In the single-phase flow, i. e., for conditions below cavitation thresholds, the mechanism of generation of pressure fluctuations is important to predict flow-induced noise/vibrations and flow/structure interactions. Nevertheless, in the presence of a vapor phase, it is crucial to (i) quantify parameters affecting the turbulent topology of the flow and (ii) to measure the pressure fluctuations due to the vortex shedding 
to characterize interaction between cavitation and turbulence. These are the aims of the present contribution.

In the context of the global effort concerning space turbo-pump development, work has been devoted to study the flow in the real inducer geometry [4]. The global behavior was characterized, and performance of the inducer was estimated for different cavitation levels, with an observation of the vapor distribution on the blades [5]. However, the turbulence in the flow and the dynamics of the cavitating structures could not be characterized mainly due to technical difficulties in performing measurements on a rotating device with a very complex geometry, such as encountered in a space turbo-pump inducer. It should be noted that two-dimensional laboratory geometries (such as Venturi) have been tested and designed to reproduce the wall pressure field existing on the suction side of the inducer's blade [6-8]. These laboratory geometries led to a better understanding of the vapor dynamics (occurrence of a re-entrant jet, periodical detachment of the rear part of the cavity, downstream convection of vapor clouds until collapse and periodic interface destabilization), but the unsteady behavior of the flow structures associated with turbulence instabilities remains poorly understood. Studies concerning this latter goal were performed in cavitating mixing shear layer $[9,10]$. The main results concerning the flow structure is that cavitation adds additional velocity fluctuations and modifies the sizes of the coherent vortices. Following pioneering work from Stutz and Legoupil [11] and Coutier-Delgosha et al. [8] in the context cavitation research in, respectively, a Venturi-type test section and on a plano-convex hydrofoil, Aeschlimann et al. [10] used x-ray attenuation to investigate a turbulent cavitating shear layer. They notably extracted the specific length scale of cavitating structures at high void fraction, highlighting the physical mechanism of the additional turbulent agitation created by the vapor phase. Consequently, in the current research, the choice of studying a fundamental case has been made to reproduce the specific dynamics of the backflow inducers in a framework allowing the identification and characterization of underlying mechanisms. Access to the gas phase information, and in particular to the void fraction field, is paramount to the development of models, scaling laws and numerical simulations. Unlike other multiphase flows where this phase is dispersed, cavitation presents high values of void fraction overall, with drastic spatiotemporal fluctuations. Measurements relying on classical approaches are challenging if not impossible, as has been stated in the past numerously. To tackle such dense multiphase flows, x-ray imaging has presented itself as a very good candidate, as extensively analyzed in a recent review [12]. Its use for cavitation research remains very sparse and it was not applied to the canonical case of the backward facing step. Besides the pioneering work cited above, recent work was conducted using time-resolved x-ray densitometry in several configurations in the lab [13,14]. Khlifa et al. [15], Zhang et al. [16] also used synchrotron x-ray phase contrast imaging to study high speed cavitating flows using a small size Venturi-type test section at the Advanced Photon Source of Argonne National Laboratory.

Compared to a mixing layer, added flow complexity lies in the presence of the separated reattaching shear layer and the recirculation area. As stated, backflow conditions are highly relevant to turbomachinery $[17,18]$. The backward facing step flow is a well-known test case in single-phase conditions and is considered as a benchmark for numerical simulations [2,19] but a nonexhaustive literature review highlights the lack of both experimental and numerical investigation in the two-phase flow regime. The present research focuses on simultaneous pressure and void fraction measurements. Combined with high-speed visualizations, they help better understand the physical mechanisms involved in the modifications of the flow by the vapor phase. Cross-correlations between the time series of the wall pressure measurements and high-speed x-ray attenuation measurements have been performed to characterize the vapor phase dynamics and the topology of the two-phase flow. The backward facing step flow, with a fixed step height and mean inlet velocity, leads to a fully developed turbulent flow (Reynolds number of about half a million), and we vary the cavitation intensity by change of the upstream pressure. The present paper is organized as follows: Sec. II is devoted to the presentation of experimental device, flow configuration and instrumentation. In Sec. III, results of the vapor phase topology and dynamics are discussed. The last section presents the conclusions. 

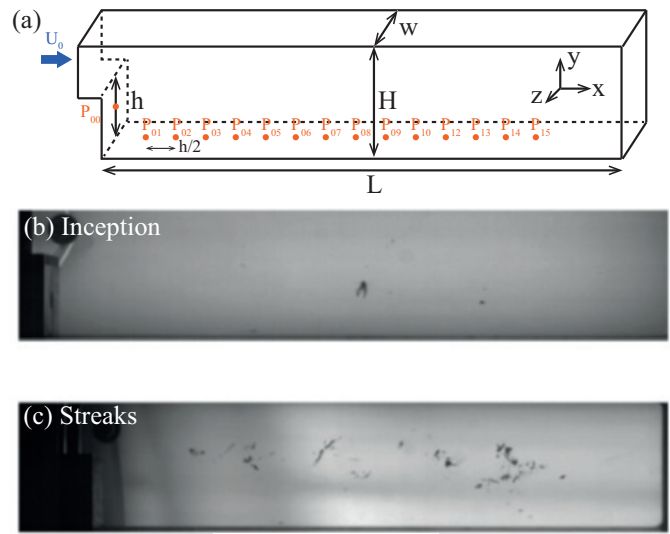
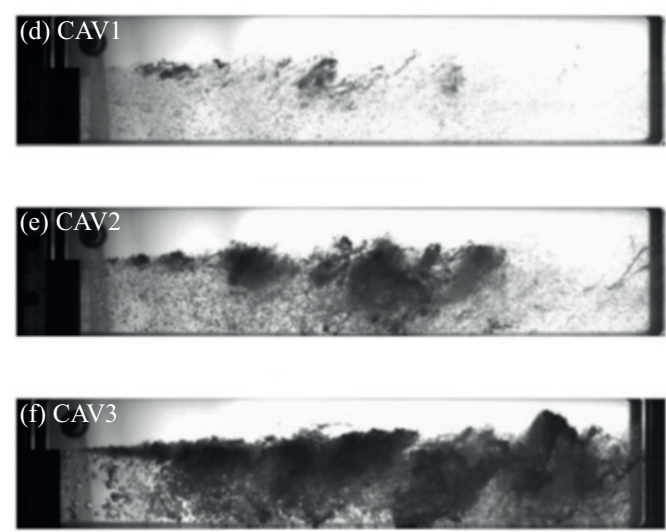

FIG. 1. (a) Backward facing step test section. The dimensions are $h=51.8 \mathrm{~mm}, w=80 \mathrm{~mm}, H=88.8$ $\mathrm{mm}$, and $L=500 \mathrm{~mm}$. The orange dots indicate the locations of the pressure probes. P00 is located the step vertical wall, at midheight; P01 to P15 re located after the step, on the test section bottom wall, spaced by h/2. (b)-(f) Representative snapshots of the different cavitation level imaged by a back-lit high-speed camera (see Table I; the paper focuses on operating conditions CAV1-3).

\section{EXPERIMENTAL METHODS}

\section{A. Two-phase backward facing step flow}

The backward facing step (sketched of Fig. 1) is placed in the test section of a hydrodynamic flow loop where the rate was fixed to $\mathrm{Q}=33 \mathrm{l} / \mathrm{s}$ provided by a $15-\mathrm{kW}$ hydraulic pump corresponding to the inlet mean velocity $U_{\mathrm{o}}$ of $11.15 \mathrm{~m} / \mathrm{s}$ in the test section before the step, leading to a fully developed turbulent flow. The step height is $\mathrm{h}=51.8 \mathrm{~mm}$ and the Reynolds number based on the step height and inlet mean velocity is $R e_{h}=\frac{U_{0} h}{v}=577000$, where $v$ is the kinematic viscosity of water. We introduce the nondimensional frame of reference $\left(x^{*}, y^{*}, z^{*}\right)$ using the step height $\mathrm{h}$. The channel width and height are $\mathrm{w}=80 \mathrm{~mm}$ and $\mathrm{H}=88.8 \mathrm{~mm}$, while its length is $L=500 \mathrm{~mm}$.

The conditions for cavitation (corresponding to the two-phase flow configurations) were controlled by the difference between a pressure reference in the inlet section and the vapor pressure of the fluid. The cavitation number $\sigma$ is defined as

$$
\sigma=\frac{P_{\mathrm{ref}}-P_{\boldsymbol{v}}}{\frac{1}{2} \rho_{w} U_{0}^{2}},
$$

where $P_{\text {ref }}$ is the pressure at the entrance of the converging section that is upstream of the test section, $P_{v}$ is the saturated pressure of water at the operating condition, and $\rho_{w}$ is the density of water. Six operating conditions were studied with variable level of cavitation, from single-phase flow to dense two-phase flow, with the following abbreviations noncav, inception, streaks, CAV1, CAV2, and CAV3. The values of the cavitation number are listed on Table I and representative snapshots are shown in Figs. 1(b)-1(f). The abbreviations follow the standard nomenclature of cavitation research with noncav standing for noncavitating flows, inception when isolated cavitation nucleation appears in the shear layer, streaking when the bubbles are grouped together in small regions of the spatiotemporal domain, and cavitation when large bubble clouds are always observable, with the intensity $1-3$ indicating increasing void fractions.

Temperature measurement of the water was required to calculate the vapor pressure and to accurately compute the cavitation number. The recorded water temperature varied within the range of $15^{\circ} \mathrm{C}$ to $25^{\circ} \mathrm{C}$ depending on operating and atmospheric conditions. The concentration of dissolved gas inside the water plays a major role in cavitation inception [20]. The experimental apparatus allowed us to measure but not to directly control for the dissolved gas concentration. The dissolved 
TABLE I. Two-phase flow conditions for different levels of inlet pressure $P_{\text {ref }}$, leading to different cavitation numbers $\sigma$.

\begin{tabular}{lcr}
\hline \hline Cases & $P_{\text {ref }}\left(10^{4} P a\right)$ & $\sigma$ \\
\hline Noncav & 13.97 & $2.200 \pm 0.019$ \\
Inception & 10.73 & $1.736 \pm 0.015$ \\
Streaks & 9.261 & $1.489 \pm 0.013$ \\
CAV1 & 8.262 & $1.325 \pm 0.012$ \\
CAV2 & 7.682 & $1.235 \pm 0.011$ \\
CAV3 & 6.423 & $1.107 \pm 0.009$ \\
\hline \hline
\end{tabular}

$\mathrm{O}_{2}$ concentration was acquired with an Orbisphere $\mathrm{MOCA}_{2}$ probe. A degasification protocol was established to reach a minimum value of $2 \mathrm{ppm}$ for each operating point, insuring reproducible operating conditions.

At cavitation number slightly below 2 (at cavitation inception case), the vapor in the backward facing step flow is created in longitudinal vortices called streaks, which then migrates to the shear layer. They form two-dimensional vortices, developing between the primary Kelvin-Helmholtz spanwise vortices. For an operating point corresponding to inception and streaks cases, the void fraction is very low, and back-lit imaging remains suitable for their study. This paper focuses on higher void fraction operating conditions (CAV1-3), where strong cavitation phenomena can lead to phase coupling mechanisms and $\mathrm{x}$-ray high-speed imaging becomes a natural candidate.

\section{B. Pressure measurements and visualizations}

The pressure measurements were performed using 16 microphones. Fifteen PCB 112A22 sensors with a resolution of $7 \mathrm{~Pa} / \mathrm{mV}$ were placed on the bottom wall of the hydrodynamic tunnel, spaced by $h / 2=25.9 \mathrm{~mm}$, and one PCB106B with resolution of $0.7 \mathrm{~Pa} / \mathrm{mV}$ was placed in the step wall at midheight as sketched as in Fig. 1(a). Two types of acquisition were undertaken. The first one was an acquisition over $5 \mathrm{~min}$ at a sample rate of $f_{s}=25.6 \mathrm{kHz}$ to compute short- and long-term statistics. The second one was an acquisition during $1 \mathrm{~s}$ synchronized with a high-speed camera with a frame rate of $4 \mathrm{kHz}$. This was done using a signal synchronizer to force simultaneous measurements, to identify the effect of cavitating vortex shedding topology on the pressure signals. In this acquisition protocol, the relative accuracy on the pressure measurement was $0.7 \%$.

Besides the high-speed x-ray attenuation measurements described below, qualitative high-speed imaging was also undertaken. The light source used to obtain back-lit imaging was composed of two continuous spots used with a diffusion device to homogenously illuminate the region of interest [see Figs. 1(b)-1(f)].

\section{X-ray attenuation measurements}

X-ray attenuation measurements were performed to obtain two-dimensional (2D) instantaneous void fraction maps and projections of the $3 \mathrm{D}$ field along the test section width. The mean and fluctuating values of the void fraction were investigated and analyzed to characterize the void fraction topology in the cavitating backward facing step flow. High-frequency measurements of void fraction inside the mixture have been performed leading to a characterization of the dynamics of the void fraction, using, for instance, spatiotemporal diagrams, and cross-correlations between void fraction fields and wall pressure signals.

\section{X-ray setup}

The x-ray generator was an industrial MCN 165 Philips tube working at a power of $3 \mathrm{~kW}$, with a voltage of $160 \mathrm{kV}$ and a current of $18.75 \mathrm{~mA}$. An x-ray image intensifier Thomson TH9432HX 


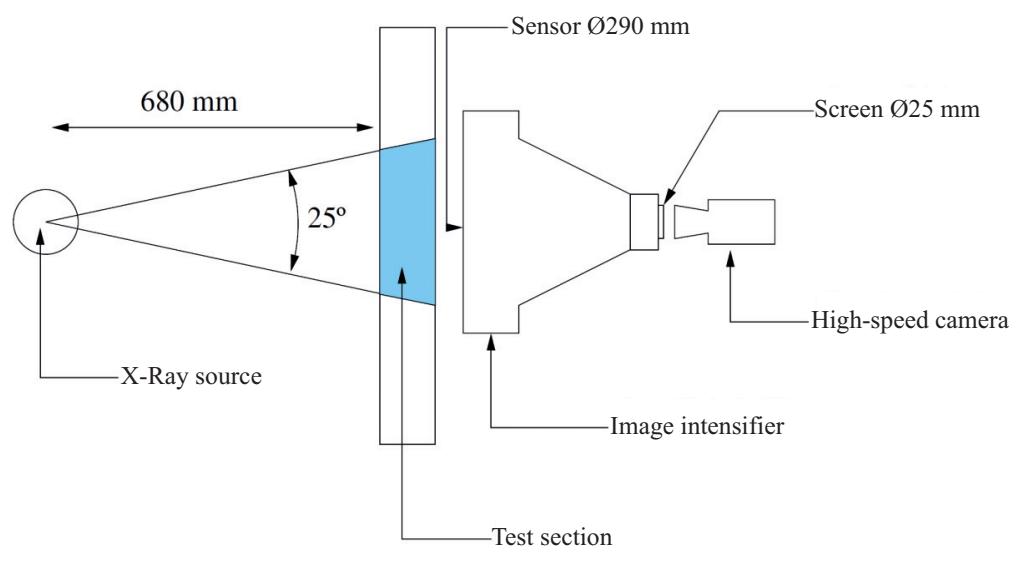

FIG. 2. Experimental setup for high-speed x-ray attenuation measurements.

was used to convert and amplify the x-ray flux to visible light, imaged by a Phantom Miro 310 high-speed camera with a framerate of $4 \mathrm{kHz}$ and a 12-bit dynamical range. The spatial resolution of the high-speed camera was $1260 \times 436$ along the $(x, y)$ plane. The number of images recorded per movie was 4096 which corresponds to an observation time of $1.024 s$ seconds. This is sufficient considering the spatiotemporal scales of the backward facing step flow. For each condition, five movies were acquired to reach good statistics.

The $\mathrm{x}$-ray source is placed on one side of the test section, while the intensifier and camera are on the opposite side, similarly to back-lit imaging configurations. These yields measurements integrated over the beam path, i. e. over the test section width. The source presents a cone angle of $25^{\circ}$, so each pixel on the $2 \mathrm{D}$ sensor effectively corresponds to the intensity of an $\mathrm{x}$-ray beam traversing the flow with an angle ranging from 0 (perpendicularly, along the $z$ axis) to $12.5^{\circ}$. Considering that the intensifier imaging diameter was $290 \mathrm{~mm}$, two imaging windows, staggered along the streamwise direction, have been introduced to cover the entire flow region. The measurement system is presented in Fig. 2.

\section{Principle}

The measurement principle is based on absorption of x-rays. For a given energy, the BeerLambert law, relating the incident x-ray intensity $I_{0}$ to the attenuated intensity $I(t)$, is given by

$$
I(t)=I_{0} \exp \left(-\mu_{0} \rho x(t)\right),
$$

where $\mu_{0}$ is the medium attenuation coefficient $\left[\mathrm{m}^{2} / \mathrm{kg}\right], x$ is the length traversed by the incoming X-ray beam $[\mathrm{m}]$ and $\rho$ the medium density $\left[\mathrm{kg} / \mathrm{m}^{3}\right]$. In the present study the absorbent medium was a mixture of vapor and liquid water and the measurements were integrated along the entire test section thickness $(80 \mathrm{~mm}$ ). The thickness (respectively, the density) of the absorbent material can be deduced from the measurements of the intensities $I$ and $I_{0}$ assuming $\mu_{0}$ and $\rho$ (respectively, $x$ ) are known. Assuming $x$ is constant and equal to $80 \mathrm{~mm}$ (neglecting the source's slightly divergent angle), the density crossed by the $\mathrm{x}$-ray beam is given by

$$
\rho=\rho_{l}-\frac{\ln \left(\frac{I}{I_{l}}\right)}{\ln \left(\frac{I_{0}}{I_{l}}\right)}\left(\rho_{l}-\rho_{a}\right),
$$

where $\rho_{l}=998 \mathrm{~kg} \mathrm{~m}^{-3}$ and $\rho_{a}=1.225 \mathrm{~kg} \mathrm{~m}^{-3}$ are the density of liquid and air, respectively. This assumption is made valid by measuring the attenuation intensity in the case of a single-phase flow of water filling the test section. This effectively provides us with a transfer function to correct for 


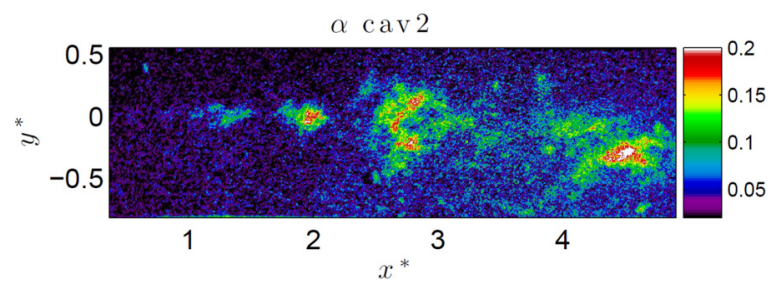

FIG. 3. Example of instantaneous void fraction field (\%) for the CAV2 conditions. The step is located at $\mathrm{x}^{*}=0$

this bias. The incident X-ray intensity $I_{0}$ is obtained following the classical procedure of taking an average image of an empty test section, correcting for the known attenuation by air. Finally, the void fraction is calculated using the density of water and vapor phase:

$$
\alpha=\frac{\rho_{l}-\rho}{\rho_{l}-\rho_{v}} .
$$

\section{Accuracy of measurements and statistical corrections}

The relative uncertainties on liquid density can be estimated using the differential density, and considering that the uncertainties on $\rho_{a}, \rho_{l}, I_{a}$, and $I_{l}$ as negligible compared to the uncertainty of $I$ measurement, as

$$
d \rho=\left|\frac{\partial \rho}{\partial I} d I\right|=\frac{\rho_{l}-\rho_{a}}{\ln \frac{I_{a}}{I_{l}}} \frac{d I}{I} .
$$

This previous equation yields the following formulation for the void fraction uncertainty:

$$
d \alpha=\frac{\rho_{l}-\rho_{a}}{\left(\rho_{v}-\rho_{l}\right) \ln \frac{I_{a}}{I_{l}}} \frac{d \tau}{\tau} \approx \frac{1}{\ln \frac{I_{a}}{I_{l}}} \frac{d \tau}{\tau} .
$$

To minimize the density uncertainty, it is necessary to maximize the contrast and integration time $\tau$. Finally, the uncertainty on exposure time is less than $0.1 \mu \mathrm{s}$, yielding an uncertainty of up to $1.3 \%$ for the measurement of void fraction.

Besides measurement uncertainty, one has to consider measurement noise. As it was observed by Aeschlimann et al. [10], noise interferences $(\beta)$ came from the measurement devices (x-ray generator + image intensifier + camera sensor). The measured void fraction can then be expressed as

$$
\alpha_{m}(x, y, t)=\alpha_{v}(x, y, t)+\beta(x, y, t),
$$

where $\alpha_{\mathrm{m}}$ corresponds to the measured void fraction, $\alpha_{v}$ being the real (noise corrected) void fraction, and $\beta$ is the noise interferences. To quantify the noise interferences, signals $\beta(x, y, t)$ were recorded with the test section full of liquid water in the absence of flow $(\alpha=0)$. The distribution of the signal $\beta(x, y, t)$ is Gaussian, with a standard deviation of about $1.8 \%$ was observed over the whole measurement area. With such a white noise, averaged void fraction was not affected by the measurement device noise, but fluctuations were slightly increased.

The x-ray measurements allowed to record image sequences and once intensity converted to void fraction and corrections applied, instantaneous 2D fields could be analyzed. An example of snapshot from the resulting movies is shown in Fig. 3.

The x-ray diagnostics highlighted the vapor phase dynamics such as large vortex structures, free shear layer instability, reattachment, wall interaction, and reverse flow for the different cavitation levels corresponding to $1 \%$ to $50 \%$ of void fraction range inside the shear layer, recirculation area, and reattachment zone. 

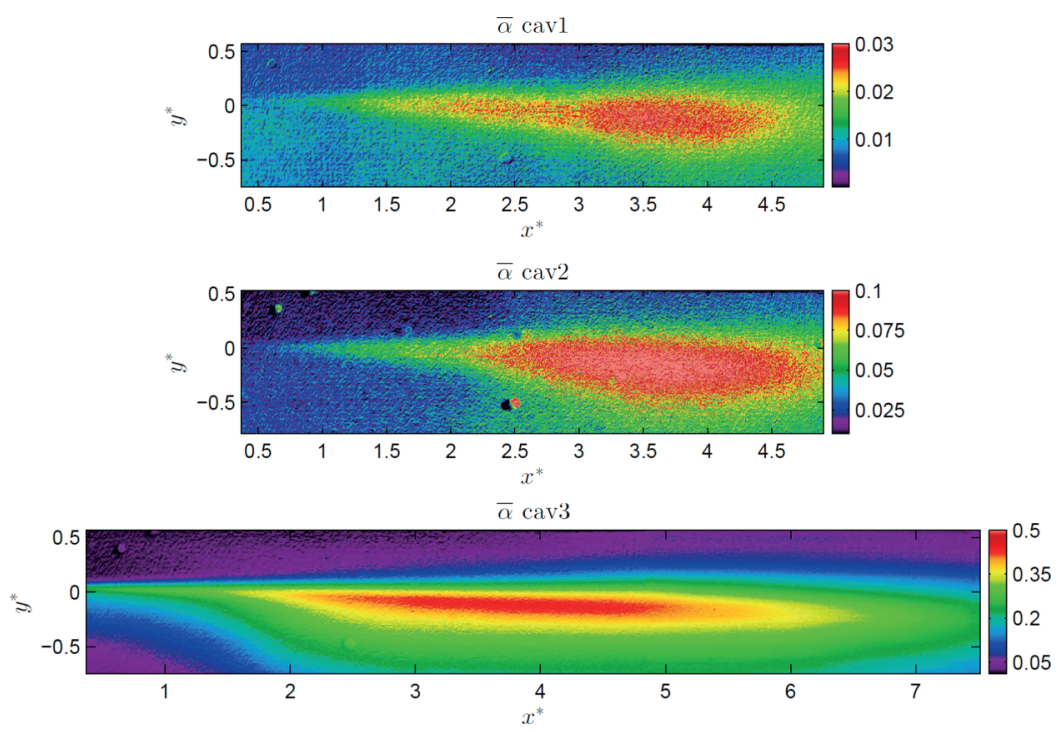

FIG. 4. Mean void fraction (\%) for different cavitation cases. Note that the colormap is different for each subfigure as the void fraction changes drastically.

\section{RESULTS AND DISCUSSIONS}

\section{A. Topology of mean and fluctuating void fraction}

Average void fraction maps were obtained by averaging the whole data set ( 5 independent series of over 4000 instantaneous maps) for each operating point. Figure 4 presents maps of the averaged void fraction for $\sigma=1.32$ (CAV1), $\sigma=1.23$ (CAV2) and $\sigma=1.1$ (CAV3). The vapor phase was contained inside the recirculation area and mixing layer. The vapor structures were condensed back to a liquid phase beyond $x^{*}=5$ for CAV1 and CAV2 and extended beyond $x^{*}=7$ for CAV3. Therefore, the CAV3 maps in Fig. 4 is computed by stitching together both acquisition windows, to be able to properly grasp the extent of the region where cavitation occurs.

While the mean void fraction field depends on the transverse coordinate $y^{*}$, global observations can be made by looking at the evolution of maximum of the mean void fraction along the longitudinal direction (see Fig. 5). This allows us to highlight three distinct areas:

(i) Vapor creation zone $\left(0<x^{*}<2.5\right)$, characterized by an increase of void fraction,

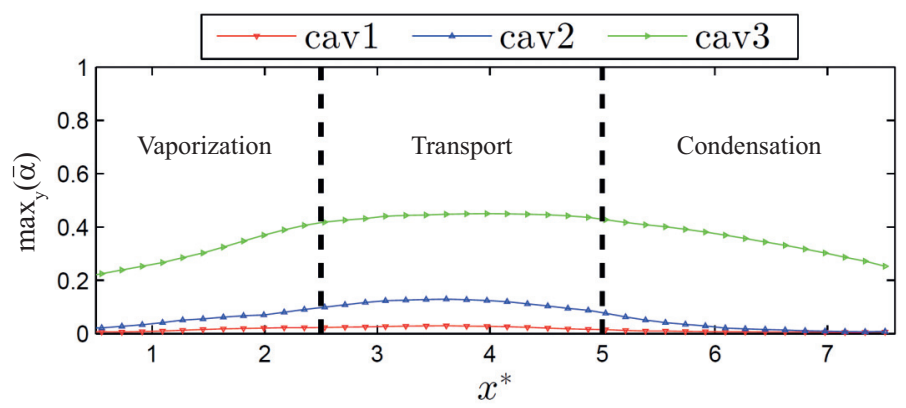

FIG. 5. Evolution of the maximum of the mean void fraction field with the longitudinal direction, for different cavitating cases. 


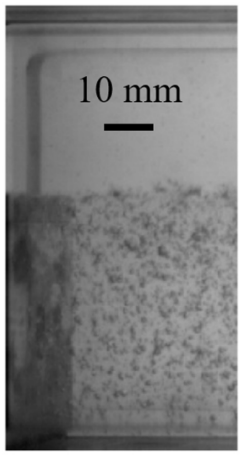

$\operatorname{Cav} 1$

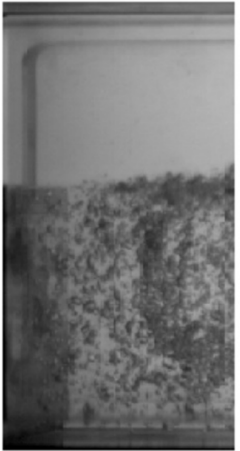

Cav2

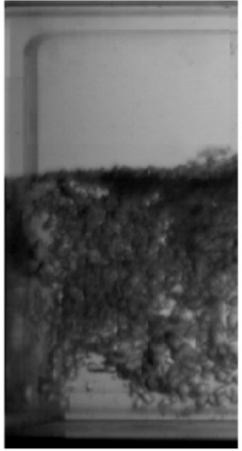

Cav3

FIG. 6. Close-up visualizations of the recirculation area, immediately after the step, for different cavitating cases.

(ii) Eddy transport zone $\left(2.5<x^{*}<5\right)$, with a constant value of $\alpha$ corresponding to the plateau of maximum value,

(iii) Condensation zone $\left(5<x^{*}<7.5\right)$, due to a pressurization of the flow associated to a decrease of the void fraction.

Note that the same distinction of the mean flow topology into three zones can be made by looking at the maximum of the mean void fraction as a function of the longitudinal distance. The latter indeed present an evolution that is qualitatively similar to the one shown in Fig. 5.

In the vicinity of the backward facing step, the void fraction for CAV1 and CAV2 remains independent of the longitudinal direction $x$ and has a low value compared to the measured values in the transport area. The vapor phase is constituted by poly-dispersed bubbles that are advected upward towards the shear layer. This area is shown on back-lit visualizations (Fig. 6) and in the movie in the Supplemental Material (CAV 3) [21].

Concerning void fraction fluctuations, maps of the rms values are presented Fig. 7. A region of high fluctuations is observed at the core of the shear layer and the maximal value evolves up to around $25 \%$ for higher cavitation level (CAV3 case), while it reaches only a few percent for the CAV1 configuration. The rms values are maximum when the mean void fraction is maximum except for CAV3 case. However, a second region with large fluctuations is also observed for this case, situated near the backward facing step $\left(x^{*}=0\right)$. This leads to a complex topology with extreme events where the rms value is approximately equal to the local mean value. One can note that moderate values of void fraction fluctuations are still observed for large values of the downstream distance $x^{*}$, even for the CAV2 case, requiring the use of both imaging windows, similarly to the CAV3 case.

\section{B. Spatiotemporal dynamics of void fraction}

Time-resolved measurements allowed the determination of spatial and temporal integral scales associated to the pressure and the void fraction and also the characterization of instabilities such as Kelvin-Helmholtz vortices, and flapping and shedding mechanisms, versus cavitation level. The spatial correlation function of the void fraction along vertical increments $R_{\alpha}\left(\mathrm{x}, \Delta_{y}\right)$ is used to estimate a characteristic length scale $L_{y \alpha}$, following:

$$
L_{y \alpha}(x)=\int_{-h / 2}^{h / 2} R_{\alpha}\left(\mathrm{x}, \Delta_{y}\right) d \Delta_{y}
$$



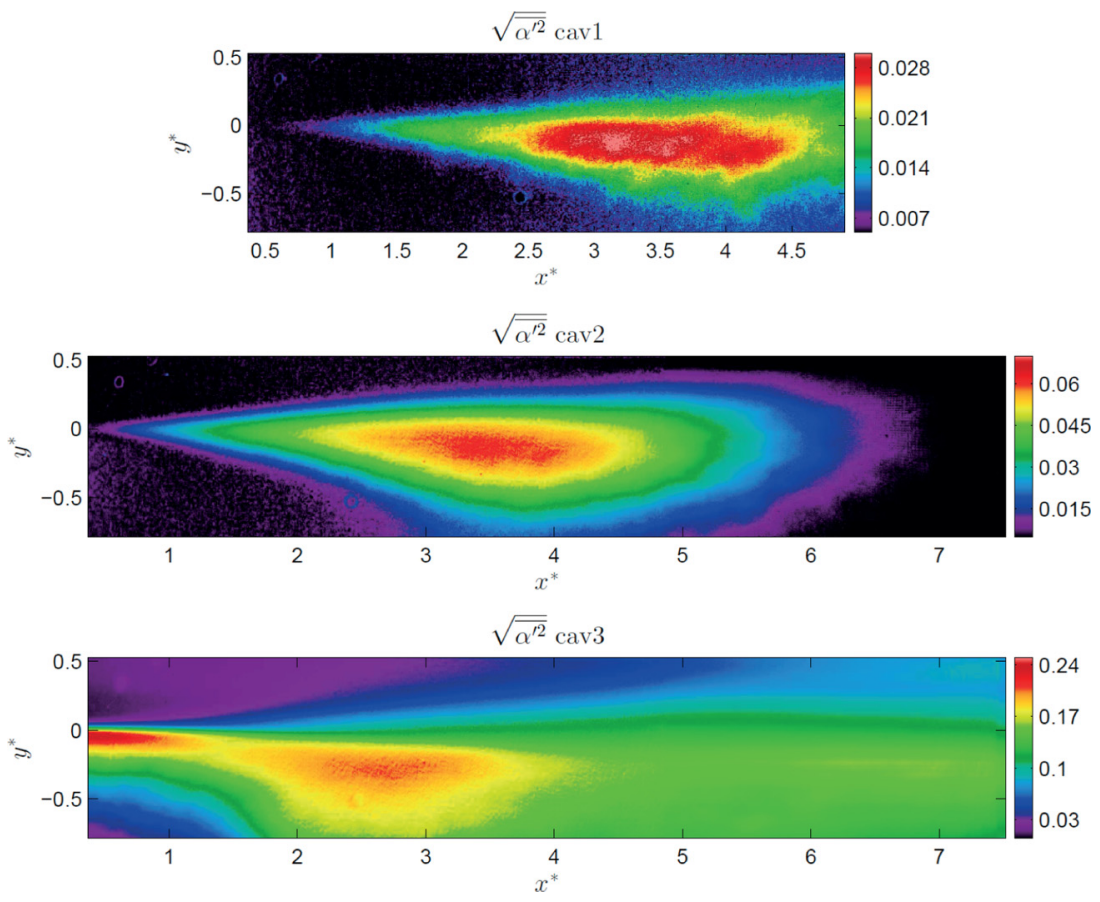

FIG. 7. Rms values of the void fraction for different cavitation cases. Note that the colormap is different for each subfigure as the void fraction changes drastically.

with

$$
R_{\alpha}\left(\mathrm{x}, \Delta_{y}\right)=\frac{\left\langle\alpha^{\prime}(x, y, t) \alpha^{\prime}\left(x, y+\Delta_{y}, t\right)\right\rangle}{\left\langle\alpha^{\prime 2}\right\rangle},
$$

where \langle\rangle corresponds to an ensemble average. The evolution with longitudinal distance of the characteristic void fraction length scale is shown in Table II, made nondimensional using the step height $h$ or the vorticity thickness $\delta_{\omega}=U_{0} / \max (\partial u / d y)$, where $u$ is obtained by particle image velocimetry. For the low cavitation level (CAV1 and CAV2 cases) the characteristic length scale, which gives an estimate of thickness of the vapor phase, is maximum in the transport area $\left(x^{*}=3\right.$ and $x^{*}=4.5$, respectively). In contrast, for the highest cavitation level (CAV3), the vapor thickness does not decrease at large values of $x^{*}$. Note that in this case, the characteristic thickness of the

TABLE II. Characteristic void fraction length-scale evolution along the nondimensional longitudinal distance for the three cavitation conditions.

\begin{tabular}{lccccccc}
\hline \hline & $x^{*}$ & 1 & 2 & 3 & 4.5 & 6 & 7 \\
\hline \multirow{2}{*}{ CAV1 } & $L_{y \alpha}^{*}$ & 0.05 & 0.2 & 0.28 & 0.24 & 0.06 & 0.03 \\
& $\frac{L_{y \alpha}}{\delta_{\omega}}$ & 0.33 & 0.66 & 0.63 & 0.4 & 0.07 & 0.02 \\
\hline \multirow{2}{*}{ CAV2 } & $L_{y \alpha}^{*}$ & 0.26 & 0.42 & 0.54 & 0.46 & 0.27 & 0.16 \\
& $\frac{L_{y \alpha}}{\delta_{\omega}}$ & 1.73 & 1.61 & 1.35 & 0.76 & 0.33 & 0.14 \\
\hline \multirow{2}{*}{ CAV3 } & $L_{y \alpha}^{*}$ & 0.58 & 0.51 & 0.52 & 0.59 & 0.65 & 0.7 \\
& $\frac{L_{y \alpha}}{\delta_{\omega}}$ & 4.14 & 2.42 & 1.36 & 1.07 & 0.89 & 0.86 \\
\hline \hline
\end{tabular}



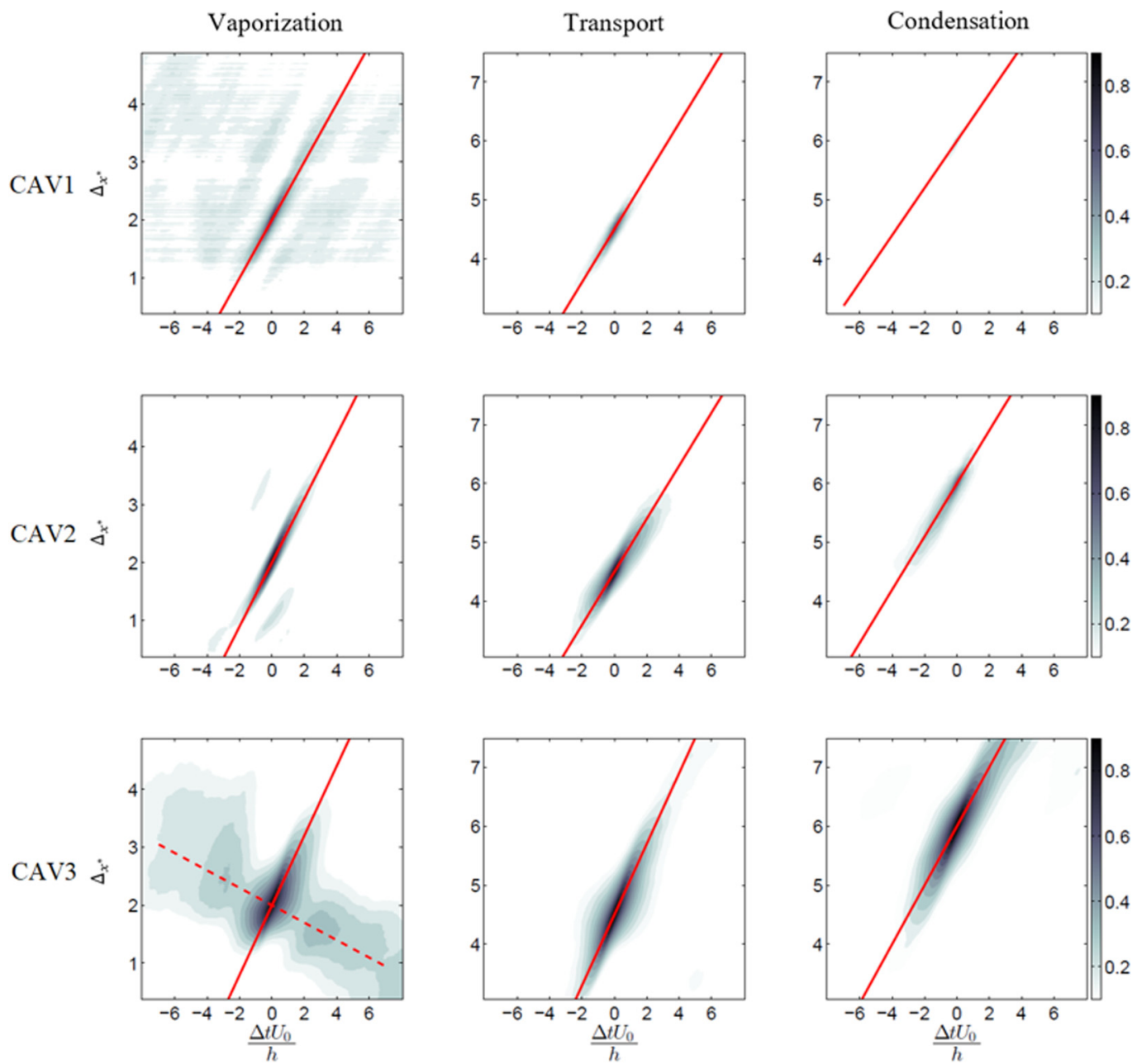

FIG. 8. Spatiotemporal correlation functions for the three developed cavitating cases. The red lines are linear fits of the correlation maxima. The dashed line is a fit of the secondary maxima.

vapor phase is always larger than half the step height, indicating that the vapor occupied a very large portion of the flow.

The spatiotemporal correlations of the void fraction have been calculated and were defined as

$$
R_{\alpha}\left(\Delta_{x}, \Delta_{t}\right)=\frac{\left\langle\alpha^{\prime}(x, y, t) \alpha^{\prime}\left(x+\Delta_{x}, y, t+\Delta_{t}\right)\right\rangle}{\left\langle\alpha^{\prime 2}\right\rangle} .
$$

The isocontours of correlation functions are shown in Fig. 8 for three cavitation cases and for specific area along the longitudinal direction (corresponding to vaporization, eddy transport and condensation areas, defined previously in Fig. 5). The spatial scale and the lifetime of each structure increase with the cavitation level with a maximum value located in the transport area. For the CAV3 case, the dynamics are slower in the vaporization area.

The red lines in Fig. 8 represent the convective velocity of vapor structures and Table III gives the values of the convective velocity of the two-phase structures. They are obtained by fitting a linear function to the local maxima of the correlation function. As the cavitation number decreases, more vapor gets created and entrained into the shear layer, lowering the fluid density, which leads to 
TABLE III. Nondimensional convective velocity values $U_{\mathrm{c}}^{*}=U_{\mathrm{c}} / U_{0}$ extracted from the spatiotemporal correlation functions.

\begin{tabular}{lccc}
\hline \hline$U_{c}^{*}$ & Vaporization & Transport & Condensation \\
\hline CAV1 & 0.5 & 0.45 & 0.4 \\
CAV2 & 0.55 & 0.45 & 0.45 \\
CAV3 & 0.6 & 0.6 & 0.5 \\
\hline \hline
\end{tabular}

an increase in the convective velocity (following Dimotakis convective velocity arguments [22]. A decrease is measured downstream in the condensation zone, associated with a structure expansion phenomenon. For the CAV3 case a secondary convective velocity is observed $\left(U^{*}{ }_{c}=-0.15\right)$ corresponding to low frequency oscillations of the vapor structure in the vaporization area. The negative value is due to pressure waves traveling upstream, created by extreme events. Recent work using time-resolved x-ray densitometry measurements to study the cavitation cloud behind a wedge uncovered the existence of bubbly shocks responsible for large-scale periodic cloud shedding [13]. These shock waves can be observed to propagate upstream on instantaneous void fraction fields. Whether the observed upstream pressure waves correspond to the same mechanism remains an open question. While not measured here, the speed of sound in the mixture at high void fraction can be estimated to be around $4 \mathrm{~m} / \mathrm{s}$ from the literature [23], which despite being of the same order of magnitude, is still slightly larger than the waves' speed, of about $1.7 \mathrm{~m} / \mathrm{s}$ here.

Large vortices are emitted and travel along the test section. When the cavitation cloud (generated by the collapse of these structures) appears, a fast pressure wave travels upstream and hits the step wall. This fast wave can trigger a new pair of vortices and induces a periodic vortex shedding mechanism, as mentioned by Saito et al. [24]. Figure 9(a) illustrates such an extreme event, corresponding to the large-scale $2 \mathrm{D}$ vortex shedding linked with a pair of 3D longitudinal vortices with high intensity. The movie in the Supplemental Material [21] shows the time-evolution of an extreme event, with synchronized pressure measurements and high-speed imaging. The signature of this vortex generation is visible in the pressure signal of the sensor on the step wall [Fig. 9(b)] and we observe a quasiperiodic structure corresponding to a main frequency of about $4 \mathrm{~Hz}$.

The mechanisms that generate vortices in the shear layer are strongly modified as the void fraction increases. Extreme events are indeed enhanced by the high-pressure fluctuations and create a phase coupling at large scales. This is most noticeable when looking at the void fraction power spectra, averaged along the vertical direction, as function of the horizontal direction (Fig. 10). We introduce here the Strouhal number as $S t_{h}=f h / U_{0}$. While the temporal dynamic presents events associated with Strouhal number in the range 0.2 to 1 for CAV1 and CAV2, it is dominated by mechanisms at much lower values for CAV3, in the vicinity of $S t_{h}=0.02$. This is associated with a low frequency oscillation of the vapor pocket size near the step. These oscillations are responsible for the high values of void fraction fluctuations around $\left(0<x^{*}<1,-0.15<y^{*}<0\right)$ in Fig. 7 . The Strouhal numbers corresponding to shedding events are, however, much lower than for CAV1 and CAV2 and seem concentrated in a narrower range of Strouhal numbers, from about 0.1 to 0.2. Note that this is probably due to a reduction of the primary Kelvin-Helmholtz instability frequency, which when extracted from downstream laser Doppler velocimetry measurements yields a Strouhal number of 0.27 for CAV3, to be compared to $S t_{h}=0.76$ for the single-phase flow.

Spatiotemporal diagrams of the void fraction and pressure (at the reference $y^{*}=0$ ) highlight the presence of the extreme events (Fig. 11, CAV3 case). Indeed, based on the maximum peak of the pressure, we distinguish a condensation front at the negative times. This front, associated with a pressure wave, goes upstream with a nonconstant velocity, and accelerates near the step wall. This propagation is followed by a new pair of vortices with different convective velocities. The first one is convected with a velocity larger than $0.6 U_{0}$ (continuous red line in Fig. 11), the second one with a range of velocity of about $0.2 U_{0}<U<0.4 U_{0}$ (red dashed line). The two-phase vortices 
(a)

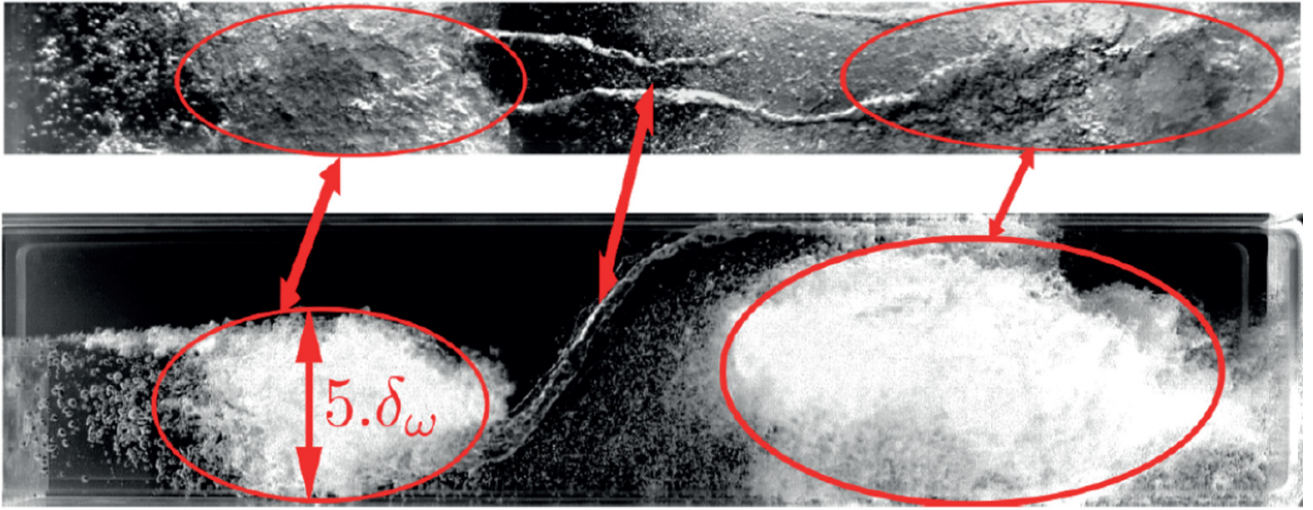

(b)
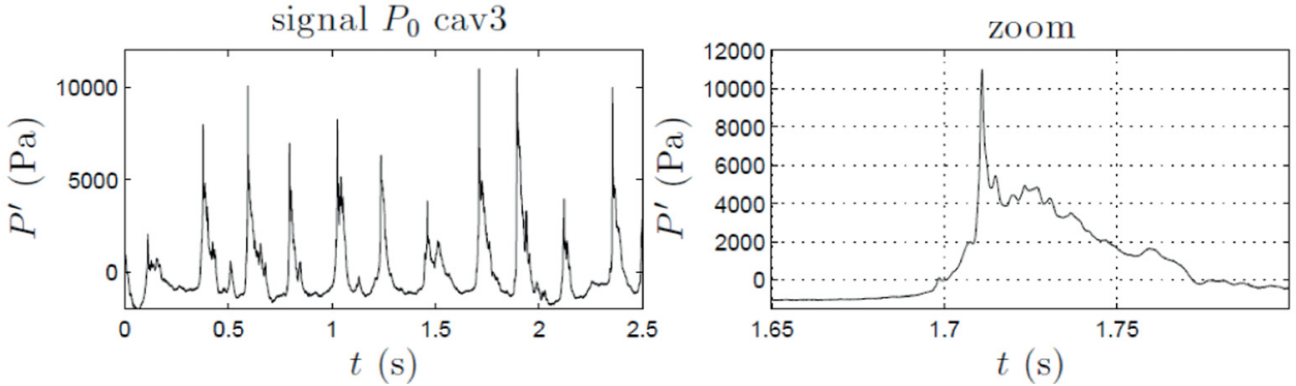

FIG. 9. (a) Extreme event in the CAV3 case (top and side views). The two longitudinal vortices are located in the center of the pictures, surrounded by two transverse vortices characterized by high void fraction and circled in red. (b) Corresponding pressure signature at the step wall.

are separated by a liquid layer corresponding to a high-pressure zone. This liquid area is convected downstream with a convective velocity in the range $0.4 U_{0}<U<0.6 U_{0}$. A similar phenomenon has been observed by Saito et al. [24] in a Venturi flow where a pressure wave is generated by a collapse of the vapor cloud inducing a re-entrant jet which then destabilizes the vapor sheet.

In our case, the pressure wave comes from downstream and condensates the fluid under the shear layer, leading to an increase of the fluid density. This phenomenon is seen in Fig. 11 between the solid and dashed red lines, for both the void fraction and pressure fluctuations. The topology of the flow passes from a shear layer mode to wake mode. They represent the two dominant modes in backward facing step flow, as was observed by Dimotakis [22] and Hudy et al. [25]. Extreme events
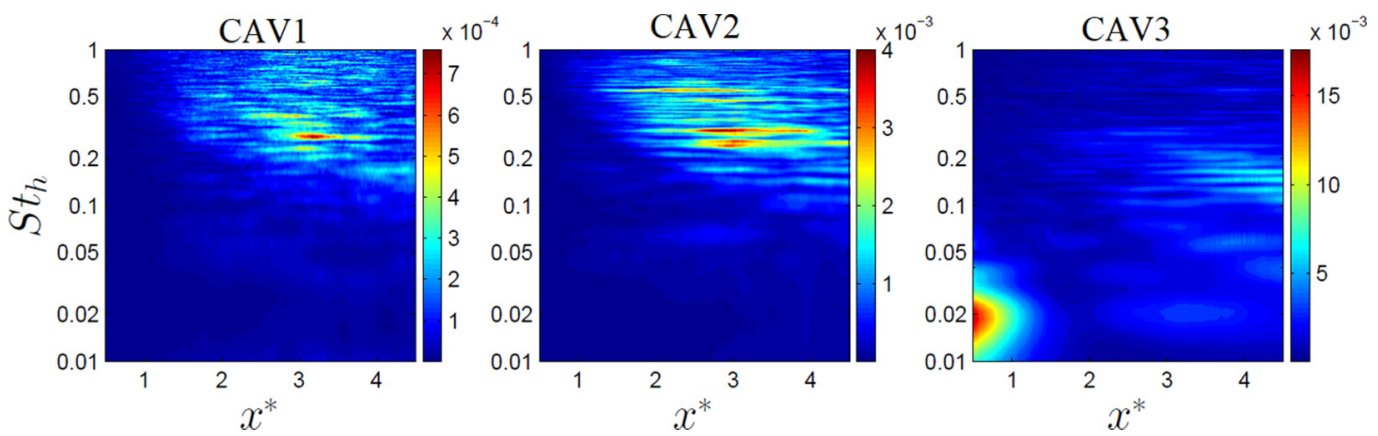

FIG. 10. Void fraction power spectra for the three developed cavitating cases. The frequency axis is normalized into a Strouhal number using the inlet mean velocity and the step height. 

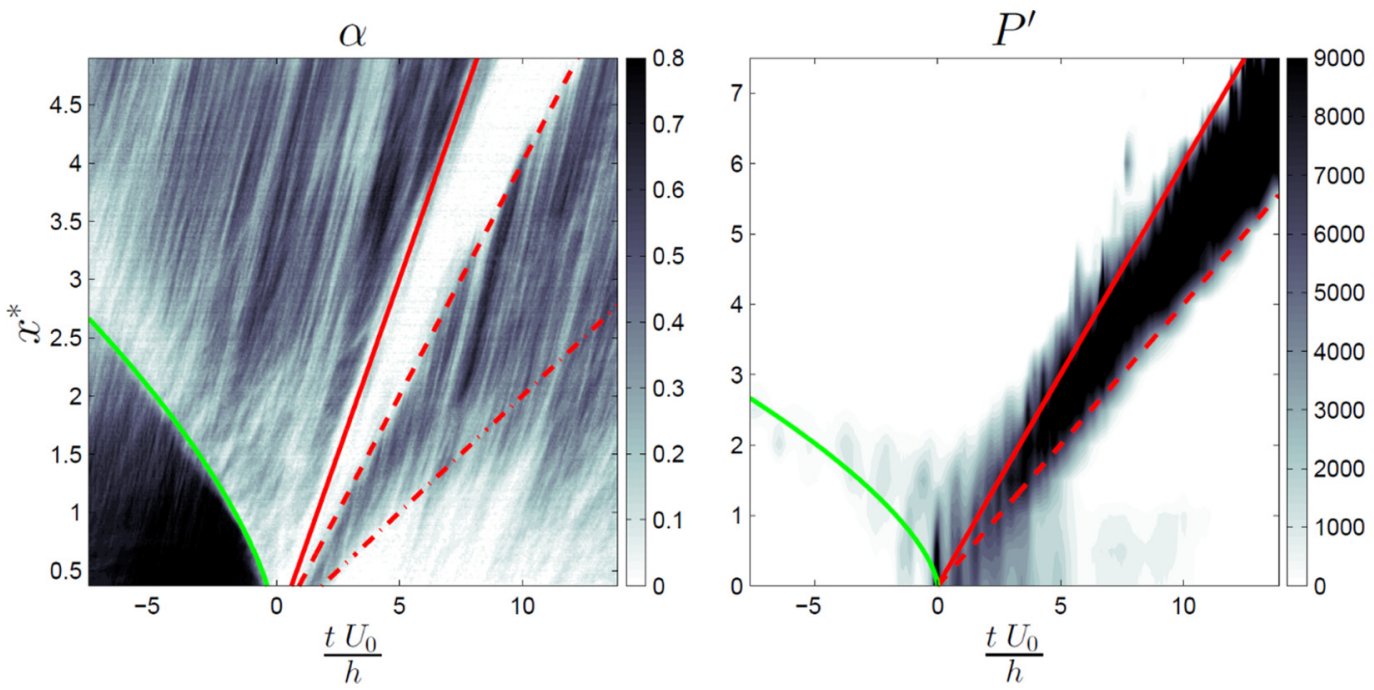

FIG. 11. Spatiotemporal diagrams of void fraction at $\mathrm{y}^{*}=0$ and pressure fluctuations $(\mathrm{Pa})$ at the step wall for the CAV3 case. The pressure diagram, obtained on 15 points along $x^{*}$, is interpolated linearly here.

at the CAV3 case correspond to an intermittency of the flow characterized by the presence of two modes of flow. The first one emanates from the dynamics of the shear layer, which entrains a fluid with low density, and the second one to a wake (see Fig. 12). As illustrated by Fig. 9(b), transition from one mode to another has a strong signature on the pressure in the test section. The pressure waves that travel upstream reach tremendous values, that collapse the cavitation cloud.

Figure 13 presents the temporal evolution of the void fraction. When the convective velocity decreases, the expansion rate of the shear layer increases, leading to the appearance of large and energetic structures where the vaporization mechanism is maximum. Vortices merge and the critical size of the vortex is reached inducing an ejection downstream. This observation clearly indicates a modification of the turbulence topology in the presence of vapor phase. The void fraction measurements, synchronized with the pressure signals, have shown that the wall pressure fluctuations are fully driven by these vortical structures.

Extreme events in the flow have been characterized by their low frequency signature and their strong correlation in space and time. Cavitation tends to amplify these events and the collapse
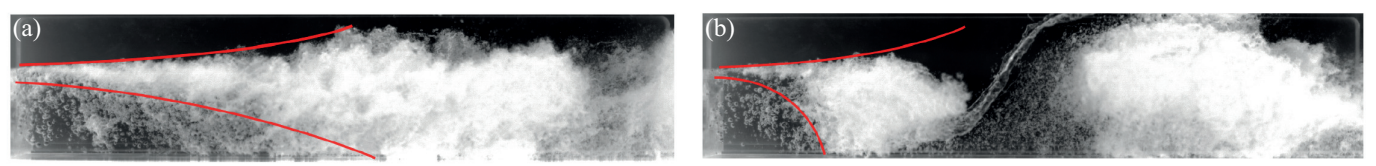

(c) Freestream
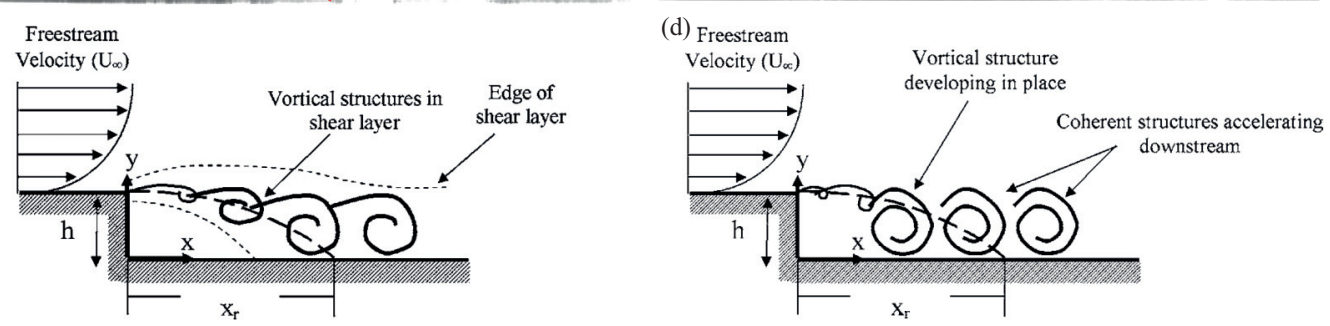

FIG. 12. (a), (b) Two instantaneous snapshots of the CAV3 case showing a shear layer (a) and wake mode (b). The red lines delaminate the shear layer expansion. (c), (d) Respective sketches from Hudy et al. 2007. 


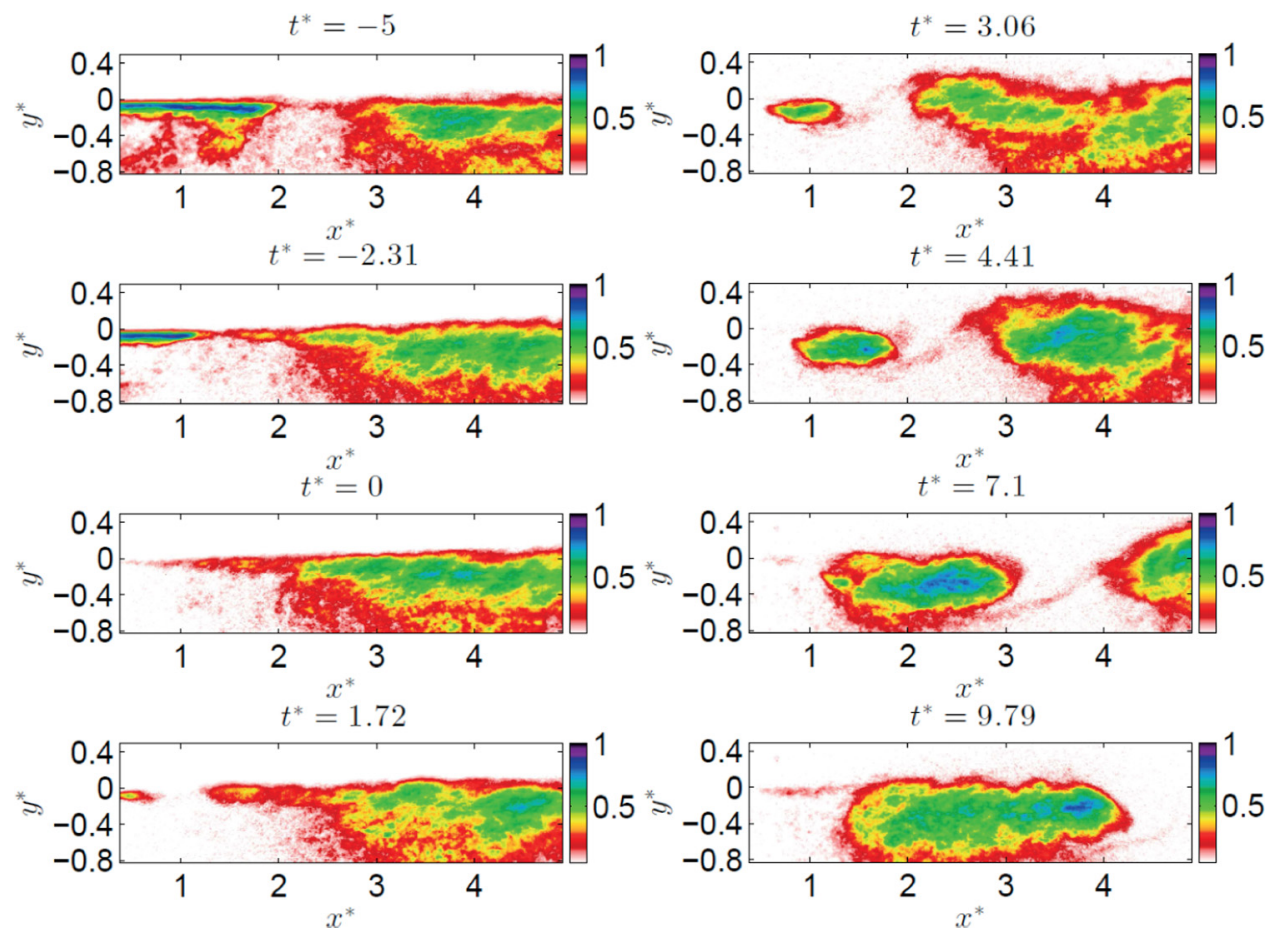

FIG. 13. Temporal evolution of the void fraction for the CAV3 case, centered around the extreme event at $\mathrm{t}^{*}=0$.

mechanism of the vapor phase leads to a periodical behavior. Moreover, this change in flow topology that has its signature on the pressure fluctuation levels and is due to the periodic apparition of extreme events, is only noticed for the CAV3 case among the operating conditions explored.

\section{CONCLUSION}

We presented an experimental study that provides an analysis of vapor distribution in a test section consisting of a cavitating backward facing step flow. High-speed x-ray attenuation measurements were undertaken for different cavitation levels, yielding time-resolved 2D void fraction fields. The void fraction measurements have been synchronized with wall pressure measurements, to characterize the effects of the vapor phase on the vortex dynamics. The mean and fluctuating topology have been characterized leading to the designation of three specific areas in the flow such as vaporization, transport, and condensation areas. Spatiotemporal correlations of the void fraction fluctuations yield convective velocities of the two-phase structures at different operating conditions, providing a data base of the cavitating backward facing step flow. At the lower cavitation number, a secondary convective velocity of negative value is observed, associated with pressure waves traveling upstream and condensing the vapor clouds. Such a high level of cavitation fluctuations leads to the modification of the topology of the flow, with phase coupling mechanisms, inducing the transition of the shear layer mode to a wake mode, creating extreme events. This behavior leads to quasiperiodic slow oscillations of both the pressure fluctuations and the void fraction. At low cavitation numbers, the void fraction remains significant downstream, allowing a growth of the shear layer vortices, followed by their core saturation. The saturation in the vortex core leads to the break-up of the recirculation cavity attached to the step and is responsible for intermittent vortex dislocation. 


\section{ACKNOWLEDGMENTS}

The authors wish to express their gratitude to the French Space Agency (CNES) and the rocket engine division of SNECMA for its continuous support.

[1] N. Furuichi and M. Kumada, An experimental study of a spanwise structure around a reattachment region of a two-dimensional backward-facing step, Exp. Fluids 32, 179 (2002).

[2] H. Le, P. Moin, and J. Kim, Direct numerical simulation of turbulent flow over a backward-facing step, J. Fluid Mech. 330, 349 (1997).

[3] I. Lee, S. K. Ahn, and H. J. Sung, Three-dimensional coherent structure in a separated and reattaching flow over a backward-facing step, Exp. Fluids 36, 373 (2004).

[4] A. J. Acosta, Y. Tsujimoto, Y. Yoshida, S. Azuma, and P. Cooper, Leading edge sweep, Int. J. Rotat. Machine. 7, 397 (2001).

[5] W. Hassan, S. Legoupil, D. Chambellan, and S. Barre, Dynamic localization of vapor fraction in turbo pump inducers by x-ray tomography, IEEE Trans. Nucl. Sci. 55, 656 (2008).

[6] B. Stutz and J. L. Reboud, Experiments on unsteady cavitation, Exp. Fluids 22, 191 (1997).

[7] S. Barre, J. Rolland, G. Boitel, E. Goncalves, and R. F. Patella, Experiments and modeling of cavitating flows in venturi: Attached sheet cavitation, E. J. Mech. B Fluids 28, 444 (2009).

[8] O. Coutier Delgosha, B. Stutz, A. Vabre, and S. Legoupil, Analysis of cavitating flow structure by experimental and numerical investigations, J. Fluids Mech. 578, 171 (2007).

[9] J. Katz and T. O'Hern, Cavitation in large scale shear flows, J. Fluids Eng. 108, 373 (1986).

[10] V. Aeschlimann, S. Barre, and S. Legoupil, x-ray attenuation measurements in a cavitating mixing layer for instantaneous two-dimensional void ratio determination, Phys. Fluids 23, 055101 (2011).

[11] B. Stutz and S. Legoupil, X-ray measurements within unsteady cavitation, Exp. Fluids 35, 130 (2003).

[12] T. J. Heindel, A review of x-ray flow visualization with applications to multiphase flows, J. Fluids Eng. 133, 074001 (2011).

[13] H. Ganesh, S. A. Mäkiharju, and S. L. Ceccio, Bubbly shock propagation as a mechanism for sheet-tocloud transition of partial cavities, J. Fluid Mech. 802, 37 (2016).

[14] J. Wu, H. Ganesh, and S. Ceccio, Multimodal partial cavity shedding on a two-dimensional hydrofoil and its relation to the presence of bubbly shocks, Exp. Fluids 60, 66 (2019).

[15] I. Khlifa, A. Vabre, Marko Hočevar, K. Fezzaa, S. Fuzier, O. Roussette, and O. Coutier-Delgosha, Fast x-ray imaging of cavitating flows, Exp. Fluids 58, 157 (2017).

[16] G. Zhang, I. Khlifa, K. Fezzaa, M. Ge, and O. Coutier-Delgosha, Experimental investigation of internal two-phase flow structures and dynamics of quasi-stable sheet cavitation by fast synchrotron x-ray imaging, Phys. Fluids 32, 113310 (2020).

[17] Y. Tsujimoto, H. Horiguchi, and X. Qiao, Backflow From inducer and its Dynamics, in Fluids Engineering Divivision Summer Meeting an Exibition (Houston) (ASME, New York, 2005).

[18] Y. Tsujimoto, Y. Yoshida, Y. Maekawa, S. Watanabe, and T. Hashimoto, Observations of oscillating cavitation of an inducer, J. Fluids Eng. 119, 775 (1997).

[19] I. Lee and H. J. Sung, Characteristics of wall pressure fluctuations in separated and reattaching flows over a backward-facing step: Part1, Exp. Fluids 30, 262 (2001).

[20] REA. Arndt, Cavitation in Vortical Flows, Annu. Rev. Fluid Mech. 34, 143 (2002).

[21] See Supplemental Material at http://link.aps.org/supplemental/10.1103/PhysRevFluids.6.044311 for a video of an extreme events (top and side view), with simultaneous pressure measurements.

[22] P. E. Dimotakis, Two-dimensional shear-layer entrainment, AIAA J. 24, 1791 (1986).

[23] H. Shamsborhan, O. Coutier-Delgosha, G. Caignaert, and F. A. Nour, Experimental determination of the speed of sound in cavitating flows, Exp. Fluids 49, 1359 (2010).

[24] Y. Saito and K. Sato, Bubble Collapse Propagation and Pressure Wave at Periodic Cloud Cavitation, in Proceeding of the 6th International Conference on Multiphase Flow, Leipzig, Germany (2007), pp. 1-8.

[25] L. M. Hudy, A. Naguib, and W. M. Humphreys, Stochastic estimation of a separated-flow field using wall-pressure-array measurements, Phys. Fluids 19, 024103 (2007). 